\title{
Determination of Reaction Pathways and Mineralogy in Material Samples using 3-D Neutron Tomography
}

\author{
V.H. DiStefano, ${ }^{1}$ J.M. LAMANNA, ${ }^{2}$ D.S. Hussey,${ }^{3}$ E.
} BALTIC, ${ }^{4}$ AND D.L. JACOBSON, ${ }^{5}$

${ }^{1}$ National Institute of Standards and Technology, Gaithersburg, MD, USA, victoria.distefano@nist.gov

${ }^{2}$ National Institute of Standards and Technology, Gaithersburg, MD, USA, jacob.lamanna@,nist.gov

${ }^{3}$ National Institute of Standards and Technology, Gaithersburg, MD, USA, daniel.hussey@nist.gov

${ }^{4}$ National Institute of Standards and Technology, Gaithersburg, MD, USA, eli.baltic@nist.gov

5 National Institute of Standards and Technology, Gaithersburg, MD, USA, david.jacobson@nist.gov

Designing new materials for sustainable industrial processes requires advanced 3-D characterization techniques to understand the internal structure of the materials and the reactions occurring at various internal interfaces. Reactions in materials can lead to material failure and degradation such as delayed ettringite formation (DEF) and alkali-silica reactions (ASR) in concrete. To accurately visualize and characterize internal structure and monitor internal reactions, nondestructive, 3-D techniques are needed to examine these new materials. The highly penetrating power of neutrons makes neutron imaging an ideal tool to measure larger material samples, including concrete cores, rock cores, and engineered materials. Neutron imaging's high sensitivity to hydrogen rich fluids also allows for direct observation of fluid/material interactions. At the NIST Center for Neutron Research, we have developed capabilities to measure structural and chemical real space information and timeresolved transportation of fluids in materials. The NeXT beamline allows for simultaneous neutron and X-ray measurements which can be coupled together to yield 3-D compositional maps with 15-20 micron resolution. Additional neutron imaging techniques are being developed to map mineral assemblages in 3-D material samples which can be used to determine strain, map 3-D crystal orientations, or determine minerology pre- and post-reaction. These techniques are important tools for in-situ studies of materials to further sustainable material development. 